\title{
BMJ Open Never events in general practice: a focus group study exploring the views of English and Scottish general practitioners of 'never events'
}

\author{
Rebecca L Morris, ${ }^{\oplus 1,2}$ Sudeh Cheraghi-Sohi, ${ }^{1,2}$ Paul Bowie, ${ }^{3}$ Aneez Esmail, ${ }^{1,4}$ \\ Carl de Wet, ${ }^{\circ 5}$ Stephen M Campbell ${ }^{1,2}$
}

To cite: Morris RL, CheraghiSohi S, Bowie P, et al. Never events in general practice: a focus group study exploring the views of English and Scottish general practitioners of 'never events'. BMJ Open 2019;9:e028927. doi:10.1136/ bmjopen-2019-028927

- Prepublication history for this paper is available online. To view these files please visit the journal online (http://dx.doi org/10.1136/bmjopen-2019028927).

Received 3 January 2019 Revised 5 April 2019 Accepted 13 June 2019

A) Check for updates

(c) Author(s) (or their employer(s)) 2019. Re-use permitted under CC BY-NC. No commercial re-use. See rights and permissions. Published by BMJ.

${ }^{1}$ Centre for Primary Care, University of Manchester, Manchester, UK

${ }^{2}$ NIHR Greater Manchester Patient Safety Translational Research Centre, University of Manchester, Manchester, UK ${ }^{3} \mathrm{NHS}$ Scotland, Glasgow, UK ${ }^{4}$ School of Primary Care, University of Manchester, Manchester, UK

${ }^{5}$ School of Medicine, Griffith University, Nathan, Australia

Correspondence to Dr Rebecca L Morris; rebecca.morris@manchester. ac.uk

\section{ABSTRACT}

Objectives To examine general practitioner (GP) understanding of the never event (NE) concept in general practice, and to identify potential enablers and barriers to implementation in UK general practice.

Design Qualitative study using focus groups. The data were analysed thematically and were informed by the normalisation process theory.

Setting General practice in Northwest England and Southwest Scotland.

Participants 25 GPs took part in five focus groups. 13 GPs were female and 12 male with an age range of 28-60. Results The NE approach of avoiding serious preventable adverse outcomes from healthcare fitted with participants expectations of the delivery of care but the implementation of strategies to prevent the specific NE was considered complex and variable. The main themes identified participants' understandings and perceived limitations of the NE concept; the embedded layers of responsibility to implement NE within practices and the work required for implementation within general practices. Participants' accounts highlighted the differential nature of work in general practice and that the implementation of initiatives to address specific NE should be situated within a learning and systems approach to implementation. Some NEs were considered more relevant and amenable to simple solutions than others which could influence implementation

Conclusions The NE concept was considered overall an important approach to help address key primary care patient safety issues. The utility of individual NEs may vary depending on the complexity of the initiatives that would be needed to manage related risks to as low as reasonably practicable.

\section{INTRODUCTION}

Patient safety is a global priority with many patient safety incidents remaining preventable. ${ }^{1}$ In response to the growing recognition of the importance of an explicit patient safety agenda, many countries, including the UK, have created national improvement strategies to reduce unintentional, but avoidable, harm to patients, for example, the Scottish
Strengths and limitations of this study

- This study explores the potential transferability and implementation of an existing secondary care patient safety policy becoming embedded into general practice.

- Complexities and unique attributes of general practice work highlighted as a potential barrier to the transferability of the never event (NE) concept.

- We recruited general practitioners with a range of practising experience and size of general practices.

- Normalisation process theory was adopted to inform the wider understanding of the context in which NE could be implemented in primary care which could be used to create tools and approaches for wider role out.

Patient Safety Programme and the Safer Patient Initiatives. ${ }^{2} 3$ The development of patient safety initiatives has received greater attention in secondary care despite primary care being a particularly important context to consider as it is where the majority of patient contacts occur. ${ }^{4}$ There have been many examples of general practice adapting approaches from other areas of healthcare and other safety-critical industries, for example, the application of a 'care bundle' approach to improve long-term condition management $t^{5}$ or the trigger review method to review patient records. ${ }^{6}$ However, the nature of work and the organisational structure within UK general practice, which is equivalent to family medicine in other countries, is notably different to hospital settings. In general practice, much of the work focuses on managing uncertainties in multiple aspects of care, for example, uncertainty in diagnosis, ${ }^{7}$ monitoring, diagnosis and treatment, and often providing self-management support. ${ }^{8}{ }^{9}$ It is unclear, therefore, due to such differences between settings and the nature of work 
contained within them, whether importing safety initiatives derived from other clinical settings will mean that their implementation will be successful and exploratory work prior to implementation should, therefore, always be conducted.

A key patient safety improvement initiative originating from the USA has been the use of so-called 'never event' (NE). Within UK the NE policy was adopted and introduced in 2009. ${ }^{1011} \mathrm{NE}$ lists have been developed and implemented for acute hospital settings in a range of national healthcare systems, including the National Health Service (NHS) in the UK, and increasingly for specific settings and clinical disciplines. ${ }^{12-15} \mathrm{~A}$ 'NE' has been defined as 'a serious, largely preventable patient safety incident that should not occur if the available preventable measures were implemented by healthcare workers. ${ }^{15}$ In hospital settings, NEs are very specific issues that are clearly identifiable using available data and preventable at an individual level with organisational implications in terms of the process of reporting and cost recovery implications. ${ }^{10}$ In general practice, this approach has the potential to offer a range of benefits, including increased awareness of important patient safety issues among the healthcare workforce and may demonstrate accountability and transparency to patients. ${ }^{2}{ }^{16-18}$ The NE process may also help develop local practice systems by prioritising incidents for significant event analysis and prospective hazard analysis approaches to minimise related risk to as low as reasonably practicable. ${ }^{19}{ }^{20}$ While uncertainty and evolving diagnoses are characteristic of the work in general practice and there are a variety of potential patient safety initiatives to improve safety, using $\mathrm{NE}$ as an exemplar may enable a more in-depth understanding of how adapting approaches from other areas is responded to in general practice and the impact it has on being adopted.
A preliminary list of 10 NEs has been developed and validated for UK general practice (see table 1) ${ }^{16}$ The NEs may be categorised as either 'errors of commission' (eg, doing something erroneously) or 'errors of omission' (ie, where there was a lapse in the quality of care). ${ }^{21} \mathrm{~A}$ recent survey to establish the frequency of occurrence of the general practice specific NEs estimated that two NEs occurred in $45 \%-60 \%$ of practices sampled, whereas three NEs were estimated to have occurred in less than $4 \%$ of practices in the last year. ${ }^{19}$ The survey identified that while NEs were likely to happen in practice there was no patterning in the types of events which were more likely to occur. ${ }^{19}$ In summary, the frequency of individual events varied across practices and events themselves. ${ }^{19}$

Previous work has illustrated that the NE concept can be translated into tangible, individual events within primary care and that these individual events are measurable. ${ }^{16} 19$ However, we do not know the views of those who would primarily be tasked with implementing and identifying the occurrence of NEs, the family doctors themselves, known as general practitioners (GPs) in the UK. The aim of this study, therefore, was to examine GPs understanding of the NE concept in general practice, as well as to identify potential enablers and barriers to implementation in UK general practice.

\section{METHODS \\ Study design}

A qualitative study design was adopted and normalisation process theory (NPT) was used as a sensitising concept for data collection and analysis. ${ }^{22}{ }^{23}$ NPT is a theory of implementation of healthcare, which focuses on understanding the work practices that enable or constrain the implementation of an intervention into routine

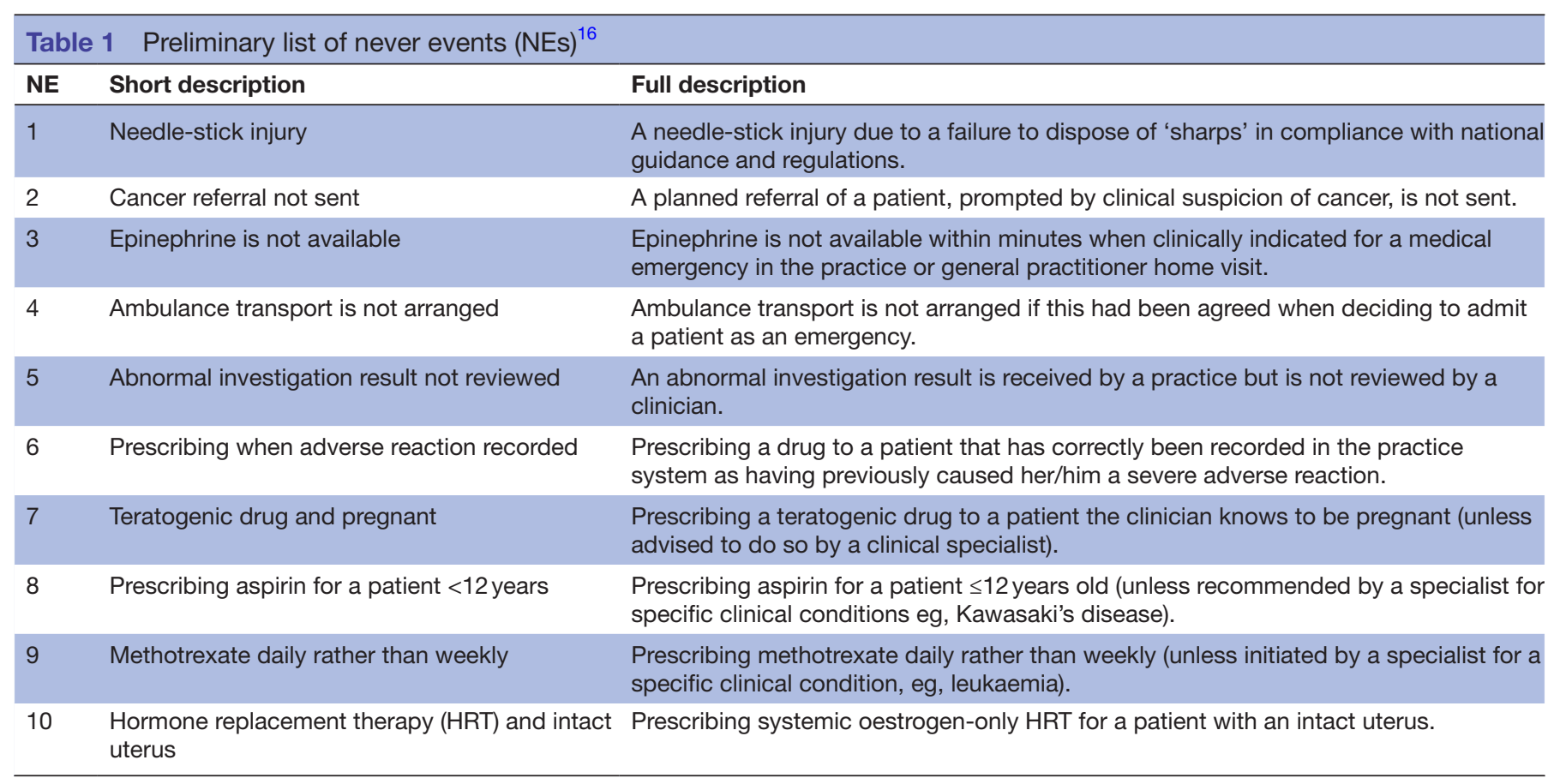


practice. ${ }^{23-26}$ By focusing on the engagement and work of implementation, it enabled us to consider the contextual, conceptual and individual factors that would be important to address before the implementation of $\mathrm{NE}$ as a patient safety intervention into everyday practice. ${ }^{23} \mathrm{NPT}$ is a sociotechnical, middle-range theory about the 'work' people do collectively and as individuals to implement and sustain complex healthcare interventions such as an NE programme. While the NE concept in the form of the preliminary list has not been implemented in routine general practice, using a theory of implementation to consider the potential enablers and barriers that would be needed to be understood to optimise implementation provides a way of making explicit the work that would be required.

\section{Sampling}

GPs were recruited using a convenience snowball sampling through existing contacts, direct approach to GPs, with support of the National Institute of Health Research (NIHR) Clinical Research Network and via NHS Education for Scotland. The focus of the study sampling was on GPs as the focus of the initial projects to develop NEs from the perspective of GPs, ${ }^{16}{ }^{19}$ and their position of usually own and manage their practices.

\section{Data collection}

Five semistructured focus groups were conducted with 25 GPs by RLM and SMC. Focus groups were conducted between April 2015 and February 2016. Two focus groups were conducted in Southwest Scotland and three focus groups were conducted in Northwest England. Focus groups were conducted in meeting rooms at either at the University, hotels or general practices. Focus groups ranged from $80 \mathrm{~min}$ to $113 \mathrm{~min}$ (average $96 \mathrm{~min}$ ). The age range of participants was between 28 and 60 (focus group 1 participant age range 43-59; focus group 2 participant age range $38-56$; focus group 3 participant age range 44-54; focus group 4 participant age range 35-42 (two participants did not give their age); and focus group 5 participant age range: 28-56) (see table 2). Focus groups were digitally recorded and professionally transcribed. Group discussions explored the participants' views on the $\mathrm{NE}$ concept content of the preliminary list, identifying strategies to prevent the listed NEs and the work required to implement them both individually and collectively (see table 3). NPT was used a sensitising concept in generating the topic guide to ensure that questions examined the four constructs of NPT. Data collection ended when data saturation was reached. Data saturation was determined when there were no new data emerging that had interpretive value. ${ }^{27}$

Each participant signed an individual consent form and completed a short demographics form before the focus group. This qualitative study adhered to and was reported using the Standards for Reporting Qualitative Research checklist. ${ }^{28}$
Table 2 Participant characteristics

No

\begin{tabular}{lc}
\hline Age (years) & 5 \\
\hline $28-40$ & 7 \\
$41-50$ & 11 \\
\hline $51-60$ & 2 \\
\hline Unknown & \\
Gender & 12 \\
\hline Male & 13 \\
\hline Female & \\
Years of experience & 4 \\
\hline $1-5$ & 5 \\
\hline $6-15$ & 15 \\
\hline $16+$ & 1 \\
\hline Unknown & \\
\hline General practitioner role & 16 \\
\hline Partner & 8 \\
\hline Salaried & 1 \\
\hline Unknown & \\
\hline Size of practice list & 2 \\
\hline Up to 3000 & 10 \\
\hline 3000-6000 & 4 \\
\hline $6001-10000$ & 8 \\
\hline 10001+ & 1 \\
\hline Unknown & \\
\hline & \\
\hline
\end{tabular}

\section{Patient and public involvement}

The initial idea was presented by RLM to members of the NIHR Greater Manchester Primary Care Patient Safety Translational Research Centre Research User Group and it was agreed that as this was a project examining GP views that public involvement was not relevant but a future study could look at the views of patients and carers. The project idea and the study design were discussed with two GP stakeholders before the study protocol was finalised.

\section{Data analysis}

Data analysis was thematic. ${ }^{29}$ Two researchers read the transcripts independently (RLM and SC-S) and inductively identified initial themes and subthemes (see figure 1). We used NPT to create a framework to examine the context of implementation and combined this with a thematic approach to examine and refine emerging themes. The themes were identified using an open approach to coding to analyse the data pertaining to the individual NPT constructs which then allowed codes to be grouped within and across constructs to form overall themes. Analysis was an iterative process informed the general inductive approach to analysis. ${ }^{30} 31$ These were then discussed, amended and/or merged until final themes and subthemes were agreed. Transcripts were then reread to ensure consistency of interpretation 
Table 3 Interview questions

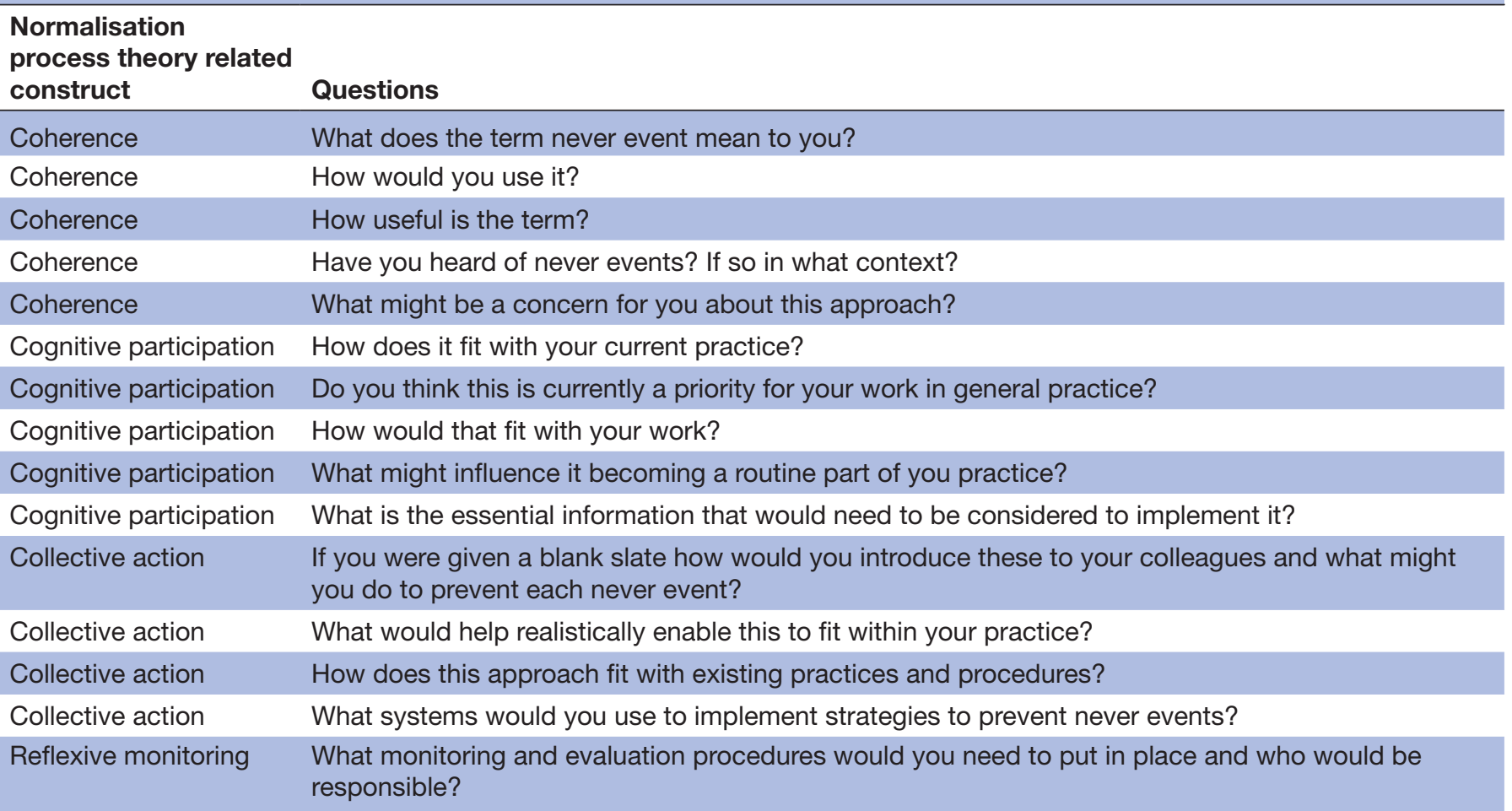

between focus groups. The key themes were circulated to all the authors for comment. The final set of themes and key themes were agreed by all authors. NPT was used within the data analysis as a sensitising concept to look at the themes that emerged from the data to consider the work involved in implementation at the level of the individual, the practice team, patients and the interface

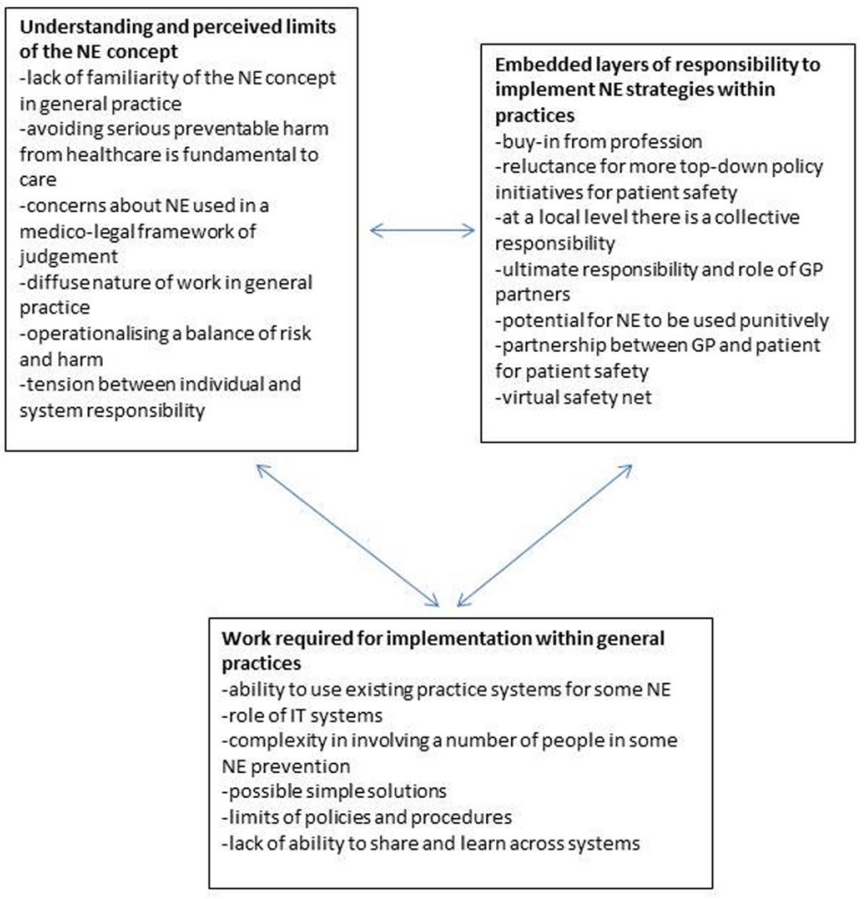

Figure 1 A diagram of the key themes and subthemes. GP, general practitioner; NE, never event. with other services across primary care or in secondary care. $^{2329}$ All data were examined across and within each focus group.

The study team consisted of two social scientist (RLM and SC-S), a health services researcher (SMC) and three academic GPs (AE, PB and CdW). Although PB and CdW were involved in the development of the initial NE list for general practice, the majority of the research team was not and the study was funded and conducted by those not involved in the development of the NE list for general practice. The composition of the research team, therefore, minimised any potential researcher bias, as there was a range of experience about the concept of NEs within the study team.

\section{RESULTS}

Twenty-five GPs took part in five focus groups (see table 2). Overall, participants expressed support for the underlying construct (ie, avoiding serious preventable adverse outcomes from healthcare) with their expectations of the delivery of care but this was within a wider of discussion of strategies to prevent NEs and the implementation of the NE list. The main themes related to participants understandings and perceived limitations of the NE concept; embedded layers of responsibility and reflexive monitoring; and the work of implementation within and against practice systems (see figure 1).

The NE concept: a hospital-centred concept so not for GPs Participants' accounts initially identified a lack of familiarity with the concept of NEs in general practice 
while simultaneously recognising their increasing use in hospital settings. Despite this, there was a general recognition that the broader underlying construct of avoiding serious preventable adverse outcomes from healthcare was valuable and fundamental to patient care, resonating with all participants approaches to patient care. However, accounts also clearly identified a consideration of a new patient safety intervention within the existing patient safety landscape and medicolegal framework of judgement. This appeared to reduce potential cognitive participation, or psychological buy-in, of some participants within a more defensive approach to medicine:

I think it's a good idea. There are things out there that you do hear of that shouldn't happen... patient safety is the most important thing. If a never event happens and it shouldn't have happened our whole ethos in medicine is do no harm, the patient always comes first...In the real world as well we all know that the lawyers are out there and the people are going to complain about things...

(FG3; R1)

Participants identified that the NE concept was used in hospital settings where they identified a potential for more immediate harm and noted the different nature of the work that characterises hospital care versus general practice which could influence engagement with the NE approach.

Although my experience of Never Events hospital-based are hospital lists are very much physical and generally immediate harm, rather than further down the line. So maybe they're going to be quite different in primary care.

(FG1; R1)

Participants' discussions identified an implicit working categorisation of degrees of risk and harm which built on a sense of collective professional experience. A central premise of the role of being a GP was considered to be operationalising this balance of risk or harm within a duty of care for patients. NEs were incidents that were judged to be outside the normative judgements of 'good' practice.

The legal...test, if it ties in with what a reasonable body of competent medical practitioners would have done you're okay, but if you step outside that collective experience, then it's more towards a Never Event.

$$
\text { (FG2; R2) }
$$

Most GPs, however, also clearly indicated a tension between the individual responsibilities of managing the inherent risk of practice with a systems approach to patient safety in the context of general practice.

It's the old adage in medicine, never say never, because the way we work...the fact that we're working with other human beings, things happen that sometimes are just totally out of your control.

(FG3; R1)

There was also a concern that the NE approach might evolve to be used punitively by external bodies rather than used as part of the existing learning system approach to safety which might limit the acceptability of the approach.

we bring these problems to our significant event analysis where we feel comfortable discussing them in a supportive team. If it became a NE would there be an onus on us to get external people in to have a look at where our quality is failing?

(FG5: R1)

\section{Preventing NEs in general practice: whose responsibility is it} Central to participants' discussions to the introduction of initiatives to prevent NEs was a debate about how these would be introduced and the effect this could have on buy-in within the profession.

I think, for quality improvement, part of the contract, this would be something... there is a lot of useless stuff in there... this would be better.

$$
\text { (FG1; M2) }
$$

There was a general sense of reluctance of further top-down, policy-led patient safety initiatives (such as the quality outcomes framework).

at the moment, governments that don't know what they're doing, and people love to take things forward, it will be misused by the government and they'll want it turned into QOF and stuff if you're not very careful.

(FG1; M1)

At a local practice level, participants agreed that while there was a collective responsibility for patient safety, GPs who were not a GP partner (owner) identified that their role was limited to individually preventing, reporting and managing NEs that may occur within their personal practice but their role to share and learn from NEs to shape future practice was limited.

if I did come across a significant event, I would report it to the practice and then hope that they would follow it through and do what's needed but I wouldn't know how to personally report something so that it gets flagged up and so people learn from it.

(FG4; R2)

It was agreed by participants that ultimately the responsibility for the implementation of patient safety initiatives was with the GP partners (owners) of the practice through setting up systems to support individuals.

F1: I think it's a collective and everybody has to hold the responsibility. 
M3: I think it's the partners' responsibility to make sure the systems are in place, and everything gets recorded properly.

(FG1; F1, M3)

While participants recognised that responsibility for the implementation and monitoring of NEs could occur at many levels (individual responsibility, practice level and commissioners), there was a concern about how it could be used punitively by different groups (for instance members of the public or the media).

...it is such an emotive word, because never event, it should never happen... also the public's perception of that. If we start to have to declare how many never events we've had in our practice how's that going to appear?

(FG5; R1)

The implementation of initiatives to address NEs was considered within the wider partnership between the GPs and patients. However, participants judged patients abilities to be partners based on an individual patient's capacity to be involved in shared decision-making which had implications for their expectations of patients abilities to be involved in patient safety.

Competence in the patient... And I don't just mean mental competence, I mean, like, you know, is this somebody who will just ignore it; is this somebody who's got low health expectations and will, therefore, assume you're going to do it all... You take a paternal view because there's nothing else you can do...

(FG2; R5)

The role of patients in implementing initiatives to prevent NEs was identified in part out of a necessary limit to professional responsibility as part of the virtual safety net for safer care within managing effectively a large patient list with a small general practice workforce.

You've got to delegate responsibility. I often say to my patients, look, I've got 4500 patients to look after, I cannot give you two, three, four hours of my time every day. It's just not possible.

(FG3; R1)

\section{Collectively engaging with NE: working within and against general practice systems for implementation}

The range of NEs was identified as being amenable to different types of initiatives, some of which would be able to work within or use existing practices systems (eg, information technology (IT) solutions to identify prescribing related NEs). However, the NEs on the list were not considered equal in terms the different technical or human processes that might have to be implemented to prevent them. For some NE (eg, NEs 7-9) there was a general consensus that these would be more amenable to alerts or reminders to prevent them using IT systems, although currently the design of the IT software would not necessarily support this:

R2: This is what really annoys me about IT because why shouldn't you have an IT system where, if you put on that a patient's pregnant, it tells you, hang on a minute...there's a pregnant patient you're trying to prescribe that drug for.

R1: Yeah, but it doesn't...

(FG2; R1, R2)

Conversely, other NEs (eg, NE 4) were considered more complex involving the coordination of multiple people and actions across different professional groups or as posing additional work that would not fit easily with existing practice and thus harder to implement. Initiatives identified to prevent these types of NEs involved more diffuse expectations of responsibility. For example, one participant described what happened with their experience of NE 4 (an ambulance transport is not arranged) and the breakdown in communication that led to an ambulance not being arranged and identifying simple solutions (eg, a checklist) to prevent it in the future:

Number four happened to me about a month ago... Busy in the head, too many things happening, just forgot. And then the second time...I spoke to the patient on the phone and then I phoned through to the receptionist to say, could she organise that this patient to go in, and fax the letter. And I thought, at that point, she would have arranged the ambulance but she hadn't. So it was a communication, it was assumption ... I should have a tick box, we should have a tick box.

(FG2; R1)

Conversely, some participants focused on individual actions and processes as most important in preventing NEs. For these participants, the nature of the workload in general practice would mean that simple solutions, such as checklists, would have limited impact when people are busy and as such these types of interventions could be fallible.

Some of these are actually ways of working, I mean, cancer referral, I haven't, as far as I know, forgotten to do a referral for ages, but that's because what I do is, immediately I decide to do a referral, I turn to the computer and I put a note on it... If you're that busy, you'll forget a checklist.

(FG2; R5)

While there are policies and local procedures that exist to prevent certain NEs (eg, NE 1) some participants gave examples of experiences they had where they had not been followed and the limits of any protocol in practice.

I've not actually had a needle stick injury because of it but I've noticed in a few practices I've been to, the sharps bin is full but you'll find that it's not been 
[emptied]... ...but there are measures in place to support that, that exist that are supposed to prevent that...Even though they might not necessarily be followed.

(FG4; R3)

Finally, participants highlighted a sense of a lack of ability to share experiences with GPs in other practices, lack of a collective system approach and frustrations of issues that increase their workload from the wider system. This creates a context in which there is a tension between the wider system and individual practices and practitioners.

...you tend to have your own experience and you live within your own practice and that's how you function...there isn't a collective system...

(FG1; F2)

\section{DISCUSSION}

\section{Summary of findings}

The NE concept was recognised as being derived from and more applicable to hospital settings than general practice, however, participants did recognise the support with the broader underlying NE construct of avoiding serious preventable adverse outcomes from healthcare. The practicalities of implementing preventative NE strategies into general practice were identified by participants and involved embedding responsibility for NE and other patient safety initiatives at different levels from the individual practitioner to the wider system and the work required varied from simple solutions, particularly using IT systems, to more complex approaches involving multiple people.

Much of the empirical literature involves examining the understanding of NEs in an acute care setting but the transferability of this concept into general practice is not well established. ${ }^{151618}$ The findings of this study suggest that while the underlying concept of NEs, avoiding serious preventable patient safety incidents, would be supported in general practice, the name 'NEs' could reduce the coherence and cognitive participation of GPs in practice. There was an initial buy-in to the underlying NE concept by participants when situated within a learning and systems approach to implementation, ${ }^{20}$ however, the content of the individual NEs on the list also influenced engagement. Some NEs were considered more relevant and amenable to simple solutions than others which could influence implementation. The implementation of preventative strategies for each individual NE would require consideration at multiple layers from the individual clinician, the practice staff and the patient. One key barrier to implementing an NE approach was a tension between the name of the approach, which was considered to set unrealistic expectations of individual clinicians (in particular by patients, policy-makers and the media), and a systems approach to patient safety (which inherently recognises the interacting demands, complexity, uncertainty, constraints and trade-offs related to everyday general practice). ${ }^{13}{ }^{32}$ This tension identified a limit to the transferability of the approach from secondary care to primary care.

Participants identified that to overcome barriers to implementation of initiatives to prevent NEs in general practices multiple nuanced responses would be needed. Some NEs (eg, prescribing related NEs) had a clearer, more linear causal, pathways which could similarly have logical and linear processes of implementation (eg, simple computer searches to identify prescribing NEs). Yet the ability to address these may lie outside of individual GPs or at the local level, for example, the design, usability and procurement of IT systems are a national policy issue which participants identified the shortcomings in the design of the systems would limit their ability to be used to prevent NEs. ${ }^{33-35}$ Other NEs were considered more complex to identify and implement initiatives to prevent them as they have more diffuse responsibility with multiple members of the healthcare team being responsible concurrently or involved the patient or the hospital. Solutions to address the more complex NEs were often relational in nature, such as a receptionist asking a GP a series of questions when they return from a home visit. Implementing strategies to prevent the more complex NEs would require strategies that reflected the more autonomous nature of healthcare professionals and patients in primary care than in settings such as hospitals and would require different approaches. ${ }^{36}$ The varying importance ascribed to different NEs, whether participants could readily identify optimal systems (either technological or procedural), and a learning environment which moved away from a sense that NEs might be used to punitively monitor individual practices were all considered important contributory factors to optimising implementation.

The organisational context and the operational nature of general practice potentially limited the transferability of the NE concept from secondary care. The diffuse nature of work in general practice was considered to be epitomised by the uncertainty of diagnosis and disease management over time ${ }^{37}$ and could influence the engagement and uptake of initiatives to prevent NEs particularly if this was in addition to their existing workload and without additional support. The temporal distance between a potential NE occurring and its effect may limit the utility of some of the NEs a progressive series of small events or lapses in care may need to occur for them to happen. ${ }^{36}$ The lack of ability to share learning across practices was considered to be a wider system issue that influenced the ability for practices from system-level data. This approach extends traditional concepts that have been developed for identifying and assessing patient safety in hospital contexts that have a different organisational approach. ${ }^{36}$ Taken together, the above suggests that there is a need for a shared learning approach to implementation that 
responds to the nature of work in general practice at a wider local level than the individual GP practice. To implement the NE list in practice will require systems to support individual work and a more nuanced approach to addressing individual events.

Participants highlighted a sense of initiative fatigue from the increasing work load being placed on general practice $^{38}$ and within a narrative of increasing workload which limited capacity to respond to new initiatives from national policy-makers. This was identified within a broader discussion about the lack of recognition and remuneration for their professional time spent on the work of implementing and monitoring other new initiatives. Overall, while there was support for the NE concept overall and implementing measures to prevent the occurrence of specific NEs, in particular those using technology to support their implementation, the perceived existing lack of distinction between this and other existing patient safety initiatives would limit any potential utility.

A strength of the study was to prospectively explore the implementation of a new patient safety initiative to illuminate different types of work (eg, coordination between members of the healthcare team (ie, collective action) or identify particular tasks or responsibilities) involved to engage and embed the NE initiatives into general practice. Accounts highlighted the diffuse nature of responsibility and the boundaries between professional groups that would implemented in the implication of this type of initiative. NPT was used as a sensitising tool $^{23}$ to examine implementation. NPT has at least two important 'strengths' to inform aspects of the data collection and analysis in this study. First, it was developed in the primary care setting with methodological rigour, transparency, and subsequently, validated through practical application in 'real-life' settings. ${ }^{24}$ Second, because NPT is about 'workability in practice' it can be applied iteratively to study temporal changes in perceptions, actions and outcomes. ${ }^{25}$ One potential limitation is that the study did not seek to examine the implementation of the NE into practice but rather to understand potential enablers or barriers and identify solutions before the NEs are implemented. This approach was adopted to inform the wider understanding of the context in which NE could be implemented in primary care which could be used to create tools and approaches for implementation. ${ }^{39}$ This study explored the views of GPs in two areas of the UK and might not reflect the views of GPs in other areas of the UK. The focus groups provided insight into participants understanding of the concept of NEs and identified strategies that could be used to prevent them. One limitation of the group setting may be that while participants signed consent forms and agreed to keep the group discussion confidential some participants may not have felt able to be open despite the seemingly candid nature of the discussion. Understanding these processes of implementation is important for developing future national interventions and a group discussion allowed for ideas to be raised and strengths and limitations to be identified within the group to maximise their potential utility.

This study examined GPs understanding of the NE concept and the nature of work that would be required to implement strategies to prevent them in routine practice. An important element of the work of implementation is the role of other members of the general practice team (both other healthcare professionals and the administrative team). Future work needs to examine their views on the implementation of NEs in general practice as well as patients and carers. In particular, future work with patients and carers needs to examine what they can do to optimise their role in patient safety as part of a shared systems approach to safety. One approach that may have some utility to developing a structured approach for each NE that incorporates the technical and human elements for risk management are barrier management approaches, such as Bowtie analysis, to identify barriers to prevent an incident from occurring and the factors that might lead these barriers to fail and increase the risk from occurring. ${ }^{40}$ This approach has the potential to provide an in-depth understanding of individual NE through prospective risk assessments. ${ }^{40}$ Furthermore, many of the NEs related to inadequately designed systems of work and technologies such as the computer software. Finally, managing uncertainty and the emotional impact of potentially making avoidable mistakes and in an NE context, these mistakes being made public, means that GPs may be ill equipped to deal with the potential fallout from such an initiative being introduced. The implications of this and specific needs to be explored and associated support (eg, in the form of Balint groups) and education (medical curricula through to continuing medical education) needs to be developed within this context. There is a lack of in-depth knowledge, training and methods to address some of these design issues to more effectively minimise risks and should be a focus of future research. ${ }^{41}$

\section{CONCLUSION}

This study sought to understand GPs views of the concept of NEs in general practice and the enablers and constrainers of implementing initiatives to prevent NEs from occurring in routine practice. The underlying concept of NEs was considered to fit with the delivery of care in general practice but the findings highlighted the need to identify a different name for the concept which would be more engaging for healthcare staff and to refine the list further to target those areas thought to be of greatest need. The findings highlighted the differential nature of work in general practice. Using an approach that incorporates a wider learning system approach to safety could increase engagement with the NE initiatives and align with other safety initiatives to embed the process into routine practice.

Acknowledgements The authors would like to thank all the participants who took part in the study. We would like to thank NHS Education for Scotland who supported 
this study. We would also like to acknowledge the support of the National Institute of Health Research Clinical Research Network (NIHR CRN).

Contributors RLM led the project, data collection, analysis, interpretation and drafted the manuscript. SC-S, PB, CdW, SMC and AE conceived the study. SMC conducted data collection. SC-S read and analysed all the transcripts. All authors developed the analysis and interpretation and critically contributed to the manuscript.

Funding The National Institute for Health Research Greater Manchester Primary Care Patient Safety Translational Research Centre (NIHR GMPSTRC) funded this study.

Disclaimer The views expressed are those of the author(s) and not necessarily those of the NHS, the NIHR, or the Department of Health.

Competing interests None declared.

Patient consent for publication Not required.

Ethics approval Ethical approach was obtained from the University of Manchester Research Ethics Committee (reference: ethics/15105)

Provenance and peer review Not commissioned; externally peer reviewed. Data sharing statement № additional data are available.

Open access This is an open access article distributed in accordance with the Creative Commons Attribution Non Commercial (CC BY-NC 4.0) license, which permits others to distribute, remix, adapt, build upon this work non-commercially, and license their derivative works on different terms, provided the original work is properly cited, appropriate credit is given, any changes made indicated, and the use is non-commercial. See: http://creativecommons.org/licenses/by-nc/4.0/.

\section{REFERENCES}

1. Carson-Stevens A, Hibbert P, Williams $\mathrm{H}$, et al. Characterising the nature of primary care patient safety incident reports in the England and Wales National Reporting and Learning System: a mixedmethods agenda-setting study for general practice. Health Services and Delivery Research 2016;4:1-76.

2. NHS Scotland. Welcome to the Scottish Patient Safety Programme. 2018 http://www.scottishpatientsafetyprogramme.scot.nhs.uk/ programme

3. Health Foundation. Learning report: Safer Patients Initiative, 2011:1-40.

4. Health Foundation. Evidence scan: levels of harm. 2014 http://www. health.org.uk/publications/levels-of-harm/

5. de Wet C, McKay J, Bowie P. Combining QOF data with the care bundle approach may provide a more meaningful measure of quality in general practice. BMC Health Serv Res 2012;12:351.

6. Bowie P, Halley L, Gillies J, et al. Searching primary care records for predefined triggers may expose latent risks and adverse events. Clin Risk 2012;18:13-18.

7. Alam R, Cheraghi-Sohi S, Panagioti M, et al. Managing diagnostic uncertainty in primary care: a systematic critical review. BMC Fam Pract 2017;18:79.

8. Daker-White G, Hays R, Esmail A, et al. MAXimising Involvement in MUltiMorbidity (MAXIMUM) in primary care: protocol for an observation and interview study of patients, GPs and other care providers to identify ways of reducing patient safety failures. $B M J$ Open 2014;4:8

9. Jerak-Zuiderent S. Certain uncertainties: modes of patient safety in healthcare. Soc Stud Sci 2012;42:732-52.

10. National patient Safety Agency. Never event - framework: process and action for primary care trusts 2009-10. 2009 http://www.nrls. npsa.nhs.uk/neverevents/?entryid45 $=59859$

11. NHS England Patient Safety Domain. Never events list 2015/16. 2015 https://www.england.nhs.uk/patientsafety/wp-content/uploads/sites/ 32/2015/03/never-evnts-list-15-162.pdf

12. Kwong LM. Never events and related quality measures following total hip and total knee replacement. Orthopedics 2010;33:838-42.

13. Jaryszak EM, Shah RK, Amling J, et al. Pediatric Tracheotomy Wound Complications. Arch Otolaryngol Head Neck Surg 2011;137:363-6.

14. Simpson KR. Obstetrical "never events". MCN Am J Matern Child Nurs 2006;31:136.

15. Department of Health. The "never events" list 2011/12, 2011:1-41.
16. de Wet C, O'Donnell C, Bowie P. Developing a preliminary 'never event' list for general practice using consensus-building methods. $\mathrm{Br}$ $J$ Gen Pract 2014;64:e159-67.

17. Francis R. Independent Inquiry into care provided by Mid Staffordshire NHS Foundation Trust January 2005 - March 2009. Volume I, 2010:1-455.

18. Gillen S. Procedures and training review aims to bring an end to 'never event'. Nurs Stand 2012;26:12-13.

19. Stocks SJ, Alam R, Bowie P, et al. Never Events in UK General Practice: A Survey of the Views of General Practitioners on Their Frequency and Acceptability as a Safety Improvement Approach. $J$ Patient Saf 2017;0:1-9.

20. Bowie P, McNaughton E, Bruce D, et al. Enhancing the Effectiveness of Significant Event Analysis: Exploring Personal Impact and Applying Systems Thinking in Primary Care. J Contin Educ Health Prof 2016;36:195-205.

21. Panesar SS, deSilva D, Carson-Stevens A, et al. How safe is primary care? A systematic review. BMJ Qual Saf 2016;25:544-53.

22. Murray E, Treweek S, Pope C, et al. Normalisation process theory: a framework for developing, evaluating and implementing complex interventions. BMC Med 2010;8:63.

23. Morris RL, Ashcroft D, Phipps D, et al. Preventing Acute Kidney Injury: a qualitative study exploring 'sick day rules' implementation in primary care. BMC Fam Pract 2016;17:91.

24. McEvoy R, Ballini L, Maltoni S, et al. A qualitative systematic review of studies using the normalization process theory to research implementation processes. Implement Sci 2014;9:13.

25. Morrison D, Mair FS. Telehealth in practice: using Normalisation Process Theory to bridge the translational gap. Prim Care Respir J 2011;20:351-2

26. Mair FS, May C, O'Donnell C, et al. Factors that promote or inhibit the implementation of e-health systems: an explanatory systematic review. Bull World Health Organ 2012;90:357-64.

27. Onwuegbuzie AJ, Dickinson WB, Leech NL, et al. A Qualitative Framework for Collecting and Analyzing Data in Focus Group Research. Int J Qual Methods 2009;8:1-21.

28. O'Brien BC, Harris IB, Beckman TJ, et al. Standards for reporting qualitative research: a synthesis of recommendations. Acad Med 2014;89:1245-51.

29. Ziebland S, McPherson A. Making sense of qualitative data analysis: an introduction with illustrations from DIPEx (personal experiences of health and illness). Med Educ 2006;40:405-14.

30. Braun V, Clarke V. Using thematic analysis in psychology. Qual Res Psychol 2006;3:77-101.

31. Thomas DR. A General Inductive Approach for Analyzing Qualitative Evaluation Data. Am J Eval 2006;27:237-46.

32. Vincent C, Burnett S, Carthey J. The measurement and monitoring of safety. 2013 http://www.health.org.uk/publications/themeasurement-and-monitoring-of-safety/

33. Phansalkar S, Edworthy J, Hellier E, et al. A review of human factors principles for the design and implementation of medication safety alerts in clinical information systems. J Am Med Inform Assoc 2010:17:493-501.

34. Avery AJ, Savelyich BS, Sheikh A, et al. Improving general practice computer systems for patient safety: qualitative study of key stakeholders. Qual Saf Health Care 2007;16:28-33.

35. Morris CJ, Savelyich BS, Avery AJ, et al. Patient safety features of clinical computer systems: questionnaire survey of GP views. Qual Saf Health Care 2005;14:164-8.

36. Vincent A, Amalberti R. Safer Healthcare. Strategies for the Real World. 2016 http://www.springer.com/gb/book/9783319255576

37. Danczak A, Lea A, Murphy G. Mapping Uncertainty in Medicine. London: RCGP, 2016.

38. Maisey S, Steel N, Marsh R, et al. Effects of payment for performance in primary care: qualitative interview study. $J$ Health Serv Res Policy 2008;13:133-9.

39. de Brún T, O'Reilly-de Brún M, O'Donnell CA, et al. Learning from doing: the case for combining normalisation process theory and participatory learning and action research methodology for primary healthcare implementation research. BMC Health Serv Res 2016;16:346.

40. McLeod RW, Bowie P. Bowtie Analysis as a prospective risk assessment technique in primary healthcare. Policy and Practice in Health and Safety 2017:1-16.

41. National Quality Board and NHS England. Human Factors in Healthcare: A Concordat from the National Quality Board. 2013 https://www.england.nhs.uk/wp-content/uploads/2013/11/nqb-humfact-concord.pdf 